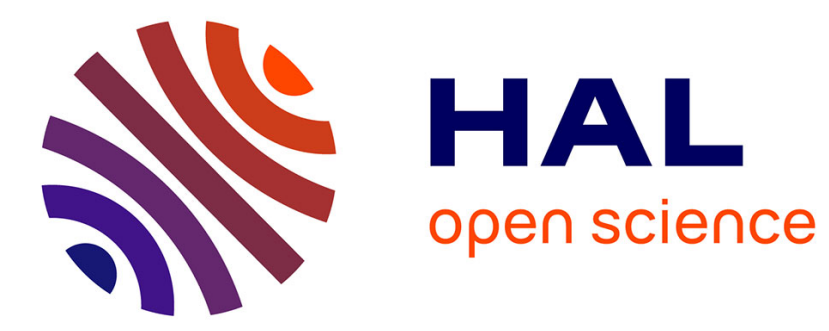

\title{
ATOMIC PHYSICS FOR HOT PLASMAS
}

\author{
L. Vainshtein
}

\section{To cite this version:}

L. Vainshtein. ATOMIC PHYSICS FOR HOT PLASMAS. Journal de Physique Colloques, 1988, 49 (C1), pp.C1-279-C1-282. 10.1051/jphyscol:1988159 . jpa-00227573

\section{HAL Id: jpa-00227573 https://hal.science/jpa-00227573}

Submitted on 1 Jan 1988

HAL is a multi-disciplinary open access archive for the deposit and dissemination of scientific research documents, whether they are published or not. The documents may come from teaching and research institutions in France or abroad, or from public or private research centers.
L'archive ouverte pluridisciplinaire HAL, est destinée au dépôt et à la diffusion de documents scientifiques de niveau recherche, publiés ou non, émanant des établissements d'enseignement et de recherche français ou étrangers, des laboratoires publics ou privés. 


\title{
ATOMIC PHYSICS FOR HOT PLASMAS
}

\author{
L.A. VAINSHTEIN \\ PN Lebedev Physical Institute, USSR Academy of Sciences, \\ Leninsky Prospect 53, Moscow 117924, USSR
}

This report treats some aspects of how to obtain and apply main atomic characteristics responsible for the intensities and satellite structures of spectral lines in hot plasmas.

\section{Plasmas in atomic physies.}

The experimental test of theoretical methods and calculated cross- sections $\sigma$ is often possible only in a plasma, especially for highly charged ions. In this case the rates $\langle v \sigma\rangle$, rather than $\sigma$, are measured for different temperature values. Another difficulty is linked to the analysis of a large number of processes which have to be taken into account simultaneously.

In laboratory plasmas (usually during an ionization stage) the ionization, excitation and dielectronic recombination rates are measured for numerous ions with $\mathrm{Z} \leq 25$. They are discussed in the report by $\mathrm{H}$. Griem at this Colloquium. Unfortunately, in some cases the results are not consistent along the isoelectronic sequence and deviate considerably (up to a factor of 1.5 - 2) from those yielded by cross-beam methods, or calculations.

Another uncertainty in the interpretation of plasma experiments is linked to the role of the secondary electron in collisions and to the possible deviations from the Maxwellian distribution function. Their inclusion requires one or more additional parameters and thus additional experimental facts.

Let us consider in more detail one example from $\mathrm{X}$-ray solar corona experiments. A possible presence of fast electrons in active regions and flares is an important point. This can be described by a distribution energy function such as:

$$
F(E)=F(E)_{\max }+A E^{-\gamma}\left(E>E_{0}\right)
$$

where $\gamma=2-3$ and $A$ is the adjusted parameter. The ionization of the main ionic component $N_{Z}>N_{Z \pm 1}$, is provided by these fast electrons if their concentration exceeds $1-3 \%$. The observed spectra of $[\mathrm{He}]-[\mathrm{H}]$ ions correspond to $A>0$, but may be simply due to an additional parameter.

Spectra in the vicinity of the resonance lines of $\mathrm{He}$ - and $\mathrm{H}$-like ions of $\mathrm{Mg}$ and Fe were obtained and discussed at the Lebedev Physical Institute. The electron temperature $\mathrm{T}$ is determined by the intensity ratio of the satellite line to the resonance line.

$$
I_{S}(j) / I_{H e}(r e s) \sim T^{-1} \exp (-\sigma E / T)
$$

However to obtain the observed intensity ratio $I_{H}$ (res) / $I_{H e}$ (res) a considerably higher temperature is required : $T_{H}>T_{S}$. At $\mathrm{T} \simeq T_{H}$ all satellites would be very faint. There is also another anomaly - the observed ratio of $q$ - and $j$-satellites, $I_{S}(q) / I_{S}(j)$, which exceeds considerably the calculated one. In an ionization equilibrium model this can be explained by direct inner-shell excitation of the q-satellite: 


$$
\left[\operatorname{Li}\left(1 s^{2} 2 s\right)\right]+e \rightarrow[\operatorname{Li}(1 s 2 s 2 p)]+e
$$

if the density of Li-like ions, $N_{L i}$, exceeds the equilibrium value $N_{L i}\left(T_{S}\right)$, i.e.

$$
T_{L i}<T_{S}<T_{H}
$$

Alternatively, both features can be explained by the presence of fast electrons ( $2 \%$ ) with $E>E_{Z}$ [He]. They increase both $N_{H} / N_{H e}$ and $\langle v \sigma\rangle$ for the q-satellite, but they do not influence the dielectronic j-satellite and the concentration $N_{L i}$. The appropriate values for the two observed ratios were calculated with one adjusted parameter A. As additional test, one can use the triplet/singlet [He] resonance lines ratio $\mathbf{G}$.

\section{The calculation of atomic characteristics.}

This is the main task of atomic physics, as far as plasma physics is concerned. There are two kinds of problems -

energies and probabilities - with quite different scales of acceptable accuracy.

2.1 Energies. For neutral and low charged atoms the theory cannot compete with experiments as concerns accuracy. The calculated energies are used to calculate probabilities and interpret experimental spectra.

For highly charged atoms $(Z-N>>1)$ the theoretical methods, at least in some cases, provide the accuracy $\Delta E / E \sim 10^{-4}-10^{-5}$, which is comparable with the experimental one in XUV and $\mathrm{X}$ regions. Two main approaches are used :

a) one based on Hartree-Fock or Dirac-Fock methods including correlation and Breit and QED corrections.

b) the method of Z-expansion (perturbation theory on Coulomb basis) with QED corrections.

The inclusion of QED is quite necessary for an agreement with experimental data : for the He-like Fe XXV resonance line, QED/E $\sim 3.10^{-4}$. Even more pronounced is the case of $[\mathrm{Be}] 2 s 2 p-2 s^{2}$ transition; for the ${ }^{3} P_{1}$ level we have the following contributions (in $10^{4} \mathrm{~cm}^{-1}$ ) :

$\begin{array}{rllll} & E_{N R} & \Delta_{E_{R}} & \Delta_{E_{s l}} & \Delta_{E_{Q E D}} \\ \mathrm{Z}=12 & 14 & 0.4 & -0.2 & -0.02(0.15 \%) \\ 26 & 34 & 9 & -5 & 0.4(1 \%) \\ 42 & 57 & 67 & -54 & 2(2.5 \%)\end{array}$

One of the problems now is the experimental test of such effects as high-order and QED correlation corrections. They might be responsible for the observed increasing of the difference $E_{t h e o r}-E_{\text {exp }}$ for Z-N $\sim 40$ in [He] and [Ne] ions, which had been observed at the Institute of Spectroscopy (near Moscow).

2.2 Calculation of probabilities and rates. We limit ourselves to some general notes on the electron-ion collisions : excitation, ionization, dielectronic recombination.

In the last years, the resonances in collisional processes and their influence on the rates, $\langle v \sigma\rangle$, were discussed in many works. These resonances correspond to bound states in the Z-1 system :

$$
\begin{aligned}
X_{Z}\left(a_{0}\right)+e \rightarrow X_{Z-1}^{* *}(\gamma) & \underset{\text { De }}{\rightarrow} X_{Z}\left(a_{1}\right)+e \\
\rightarrow & \\
\gamma=\operatorname{an\ell },(Z /(n-1))^{2} & \approx \Delta E\left(a a_{0}\right)-E
\end{aligned}
$$


The excitation cross-section is :

$$
\sigma\left(a_{0}-a_{1}\right)=\sigma_{p o t}\left(a_{0}-a\right)+\sum_{a} \sigma_{r e s}\left(a_{0}-a-a_{1}\right)
$$

and at the threshold of the channel, a

$$
\begin{array}{r}
\sum_{a_{1}} \sigma_{r e s}\left(a_{0}-a-a_{1}\right)=\sigma_{p o t}\left(a_{0}-a\right) \\
E<\Delta E \quad E>\Delta E
\end{array}
$$

Although $\sigma_{\text {res }}$ has a complicated E-dependence in a narrow energy range the average $\langle v \sigma\rangle$ (i.e. for the plasma applications) is not sensitive to details of this structure. Therefore, one can use equation (6) in place of the exact function $\sigma_{\text {res }}(E)$.

The radiative transition in $\mathrm{Eq}$. (4), i.e. dielectronic recombination, can compete with the resonant scattering. The branching ratio (of the decay probabilities) :

$$
b=\frac{W_{a i}\left(a n \ell, a_{1}\right)}{W_{a i}+W_{r a d}}
$$

is decreasing for large $n$ and $Z$; for $Z>20$ the resonances are less important. This effect was not included in some calculations.

However, this decrease means an increase of the satellite intensities, which are not always resolved in $\mathrm{X}$-ray spectra.

One can divide the various methods of cross-section calculation into two groups.

A. The I-order methods (CB, DW, ...) with exchange and normalization (Kmatrix). Resonances can be included by means of extrapolation of closed channels cross-section, according to (4).

B. A simultaneous calculation for a set of channels : - the cc method which is a solution of a set of equations; - the R-matrix method based on DW or CB functions for open channels, and a set of correlation functions taking into account the closed channels. These B-methods are more accurate, of course, yet very complicated and involve much time on big computers. Besides, in some cases it is difficult to reveal a physical reason of discrepancy in the data given by different authors.

A comparison of the results shows that more simple methods, A, provide in most cases sufficient accuracy for $\langle v \sigma\rangle$, if necessary physically clear conditions are fulfilled. In particular, the wave functions of the target and outer electrons should be cross-orthogonal in the exchange part

$$
\begin{array}{r}
<\Psi(1) \bar{F}(2)\left|\frac{1}{r_{12}}\right| \Psi^{\prime}(2) \bar{F}(1)> \\
<\Psi\left|\overline{F^{\prime}}>=<\Psi^{\prime}\right| \bar{F}>=0
\end{array}
$$

There is also the question of target wave functions, namely: 1) intermediate coupling - mixture of direct scattering amplitude in the transitions with $\Delta S=1$, with exchange amplitude, 2) configuration mixing - usually within one complex, 3) the type of radial functions - HF, TF (Thomas - Fermi), semi-empirical.

It seems most useful and most simple to use semi-empirical radial functions with asymptotic expansion corresponding to correct energy. 
Until now there have not been sufficient experimental data to test different theoretical methods. For this reason many authors use most complicated approaches. However, it is difficult to provide all necessary data in this way.

2.3 Ionization. Here comparatively simple I-order methods are being used. The results are in a sufficiently good agreement with the experiment, if one takes into account inner-shell ionization - direct and through excitation of autoionization levels. Unfortunately, this excitation is often sensitive to the target wave function. For applications to plasma it is sufficient to consider the shells with the same quantum number $\mathrm{n}$.

The dielectronic recombination (DR) is :

$$
\begin{array}{r}
X_{Z+1}\left(a_{0}\right)+e \rightarrow X_{Z}^{* *}(\gamma) \underset{\text { AI }}{\stackrel{\mathrm{DR}}{\rightarrow} X_{Z+1}^{*}\left(\boldsymbol{a}^{\prime}\right)+e} \\
\gamma=\operatorname{an\ell } L S J, \\
\alpha^{\mathrm{DR}} \sim \sum_{a} \sum_{\substack{n \ell \\
\Sigma s_{J}}} \sum_{\gamma^{\prime}} g_{\gamma} \frac{W_{a i}\left(\gamma, a_{0}\right) W_{r a d}\left(\gamma, \gamma^{\prime}\right)}{W_{a i}(\gamma)+W_{r a d}(\gamma)}
\end{array}
$$

The calculation of $W_{a i}$ is similar to that of the excitation. The main difficulty is the summing over a very big number of terms. Due to the nonlinear formula it is impossible to sum analytically over the angular momenta without additional approximations.

The detailed calculations were fulfilled recently for a number of ions. However, in most cases simple approximate methods are successfully used. Another problem with DR is linked to secondary processes. In particular,with increasing density we have

a) ionization from the level $\gamma$ :

$X_{Z}(a n l)+e \rightarrow X_{Z+1}(a)+2 e$

and also the diffuse ionization $: \mathrm{n} \rightarrow \mathrm{n}+1 \rightarrow \mathrm{n}+2 \ldots$

b) redistribution to the greater values of 1 . For large $1, W_{a i}$ becomes small, and $\alpha^{D R}$ is increasing. Thus, (a) and (b) have the opposite direction with increasing density, $N_{e}$. At first (a) is more important and $\alpha^{D R}$ increases, then (b) becomes prevailing and $\alpha^{D R}$ decreases to zero. This seems to be the reason of contradictory results of $\alpha^{D R}$ measurements in the plasma experiments.

Fortunately, in the solar corona these secondary processes are not important.

\section{References:}

1. I.I. Sobelman, L.A. Vainshtein, E.A. Yukov "Excitation of Atoms and Broadening of Spectral Lines." Springer Verlag, Berlin, Heidelberg, New York, 1981

2. R.K. Janev, L.P. Presnyakov, V.P. Shevelko "Physics of Highly Charged Ions" Springer Verlag, Berlin, Heidelberg, New York, 1985 